\title{
Anti-plane dynamic response of a non-circular tunnel with imperfect interface in anisotropic rock mass
}

\author{
Xuepeng Zhang ${ }^{\mathrm{a}, \mathrm{b}} \quad$ Yujing Jiang $^{{ }^{*} \mathrm{a}} \quad$ Satoshi Sugimoto $^{\mathrm{a}}$
}

a) School of Engineering, Nagasaki University, 1-14 Bunkyo-machi, 852-8521 Nagasaki, Japan

b) State Key Laboratory of Mining Disaster Prevention and Control Co-founded by Shandong Province and the Ministry of Science and Technology, Shandong University of Science and Technology, Qingdao 266590, China

Correspondence: Yujing Jiang

Corresponding Address:

School of Engineering, Nagasaki University,1-14 Bunkyo-machi, 8528521 Nagasaki, Japan.

E-mail: jiang@nagasaki-u.ac.jp

Tel: +81 958192612 


\begin{abstract}
In anisotropic rock mass, the dynamic response of a non-circular tunnel with imperfect interface is theoretically presented under an incidence of an anti-plane SH wave. For the anti-plane problem in anisotropic medium, three elastic constants can describe the characteristics of medium anisotropy according to the generalized Hooke's Law. The wave function expansion method and the complex function are applied to express the wave fields with three unknown coefficients. By introducing the spring elastic model, the imperfect boundary condition around the tunnel lining can be satisfied to determine the coefficients. In numerical examples, effects of rock anisotropy and imperfect interface around the tunnel lining are discussed in detail, accompanied with incident angle and wave frequency. Analytical solutions show that both rock anisotropy and interface have considerable influence on the dynamic response of underground structures, which should be paid attention to for the design and construction of deep underground tunnels.
\end{abstract}

Keywords: dynamic response; imperfect interface; rock anisotropy; SH wave; non-circular tunnel

\title{
1. Introduction
}

Knowledge of response of and potential damage to underground structures is of great importance for the design and construction of deep underground tunnels under different kinds of loadings, especially strong earthquakes. As the effective tools to tackle problems such as complicated geological condition and arbitrary shape of tunnel lining, numerical methods including the finite element method (Ren et al., 2005; Wang et al., 2014; Wu et al., 2015; Yu et al., 2016), the finite difference method (Shen et al., 2014), the boundary element method (Zimmerman et al., 1993; Yu et al., 2010; Dravinski et al., 2011) and the discrete element method (Duan et al., 2018) have been widely applied to analyze the response of underground tunnels under static overburden or dynamic loadings. Even though there is wide application of the numerical methods, analytical methods remain the convenient and efficient ways of providing the direct qualitative insights into the physical mechanism (Liu et al., 2013; Fang et 
al., 2016a). Using the wave function expansion method, varieties of studies have taken into consideration plane or anti-plane stress and displacement of underground tunnels with or without lining embedded in a homogeneous, isotropic and linear elastic full or half space (Pao and Mow, 1973; Davis et al., 2001; Smerizini et al., 2009; Lin et al., 2010; Liu et al., 2012, 2013). For a non-circular tunnel, such as a rectangular tunnel, semi-circular tunnel or horseshoe-shaped tunnel which are common in engineering applications, the complex variable method and the weighted residual method are introduced to solve the problem caused by the scattering and diffraction of the seismic wave (Wang et al., 2005; Gatmiri and Eslami, 2007; Liu et al., 2016).

As a matter of fact, the tunnel excavation, especially using drilling and blasting, may result in a very irregular and rough excavation surface. Therefore, due to the existence of micro-cracks or interstitial media, the interface between the tunnel lining and its surrounding rock mass is not always perfect. The stress and displacement behaviors of tunnel lining are very dependent on the surface status (Son and Cording, 2007). From the qualitative and quantitative point of view, the analytical results are different from the true ones if a correct interface is not placed around the tunnel lining. Recently, several imperfect interface models involving the elastic interface model (Yi et al., 2014; Fang et al., 2015, 2016a) and time-dependent visco-elastic model (Fang et al., 2016b, 2017) have been introduced.

For convenience of derivation, the rock mass is usually assumed to be isotropic. In practical engineering, due to the mineral foliation in metamorphic rocks, stratification in sedimentary rocks and discontinuities in the rock mass, many rocks have anisotropic characteristics, i.e. their mechanical, thermal, seismic, and hydraulic properties. Generally speaking, rock anisotropy causes a non-uniform stress state and displacement around the boundary of an underground opening, creates a non-uniform depth of failure and allows for stress-induced failure (near wall degradation) to possibly occur at relatively low stress levels and under unexpected stress conditions (Bewick and Kaiser, 2009). Rock anisotropy is one of the most distinct features that must be considered in rock engineering disciplines. And a few studies have been devoted to investigating the anisotropy effect (Liu, 1988; Shi et al., 1996; Han and Liu, 1997; Chen, 2012, 2015). But previous research has failed to consider the effect of an imperfect interface on the dynamic response of an underground tunnel. 
This study aims to clarify the effects of rock anisotropy and an imperfect interface on the dynamic response of underground structures. Here, the wave function expansion method and complex function are used to describe the wave fields. The mapping function is adopted to describe the horseshoe-shaped tunnel lining, the elastic spring model is used to represent the imperfect interface. Through a series of numerical results, the effects of rock anisotropy and imperfect interface on the dynamic stress concentration are examined in detail, accompanied with the incident angle and wave frequency.

\section{Computation model and governing equations}

A deep underground tunnel with horseshoe-shaped lining is embedded in a homogeneous, anisotropic, and linearly elastic rock medium. An anti-plane shear wave (SH wave) propagates into the cross-section of the tunnel lining with an incidence angle $\alpha$. Due to the geometrical condition and the character of applied loading, this problem can be simplified into a plane strain problem. A vertical cross-section of the essentially two-dimensional problem and the adopted coordinated systems are shown in Fig. 1. Two coordinates systems are introduced as one Cartesian coordinate systems $(x, y)$ in the $Z$ plane and one polar coordinate system $(r, \theta)$ in the $\zeta$ plane having a common origin at the center of the tunnel as system $(x, y)$. The $z$-axis denotes the axis of the tunnel lining, not being plotted in Fig. 1.

For an anisotropic material, 36 elastic constants are required to describe its property according to the generalized Hooke's Law. Since anti-plane SH wave is considered in the present study, only three elastic constants $C_{44}, C_{45}, C_{55}$ can describe the deformation of the surrounding rock medium. Since the strain energy density is positive, the following constraint $C_{44}>0, C_{44} C_{55}$ $C_{45}{ }^{2}>0$ should be incorporated. Besides, the density of the surrounding rock medium is characterized by $\rho_{\mathrm{R}}$. The tunnel lining with outer radius $R_{1}$ and inner radius $R_{2}$ is assumed to be homogeneous, isotropic, linearly elastic material with properties characterized by Lamé constants $\lambda_{\mathrm{L}}, \mu_{\mathrm{L}}$ and density $\rho_{\mathrm{L}}$. The imperfect interface between the tunnel lining and its surrounding rock medium is taken into consideration. 


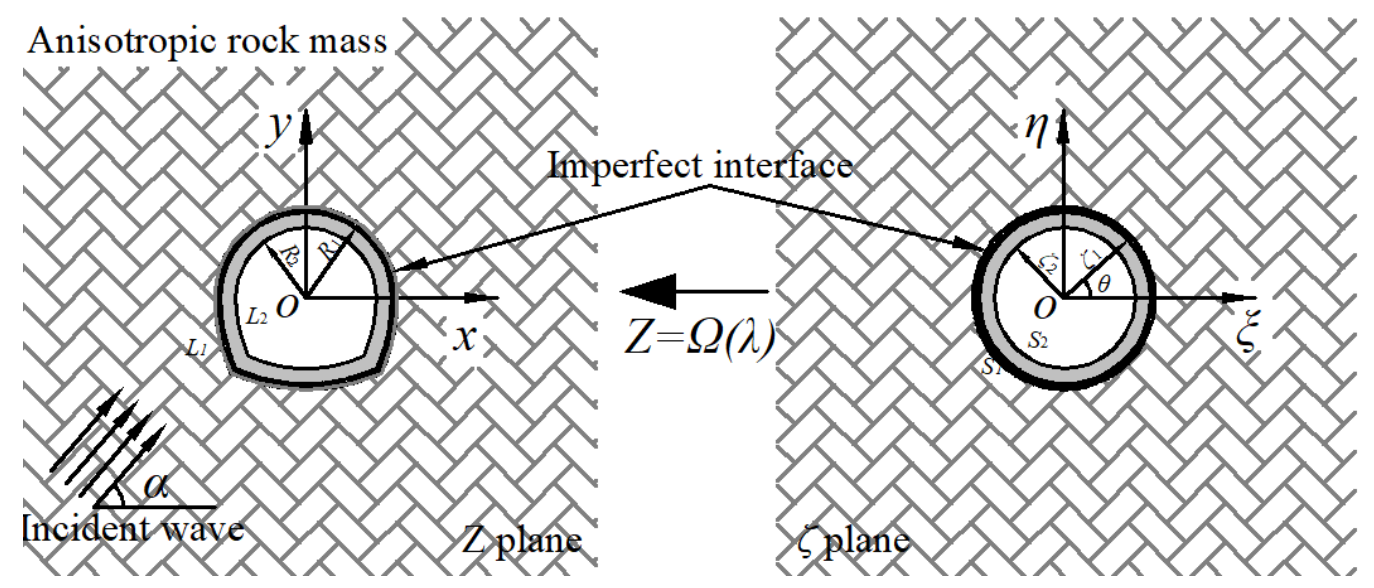

(a)

(b)

Figure 1 A horseshoe-shaped tunnel with imperfect interface under anti-plane shear wave in the $Z$ plane (a) and its mapping ring region in the $\zeta$ plane (b).

In a fixed Cartesian coordinate system $(x, y, z)$, let $u_{x}, u_{y}, u_{z}$ be the displacement in the three directions, respectively. For the anti-plane deformation, only the out-of-plane displacement $W$ is considered, that is,

$u_{x}=u_{y}=0 ; u_{z}=\mathrm{W}(x, y, t)$

in which $t$ is the time variable. The governing equation in the anisotropic mass in the $Z$ plane is (Liu, 1988):

$\left(C_{55}-C_{44}+2 i C_{45}\right) \frac{\partial^{2} W}{\partial Z^{2}}+2\left(C_{44}+C_{55}\right) \frac{\partial^{2} W}{\partial Z \partial \bar{Z}}+\left(C_{55}-C_{44}-2 i C_{45}\right) \frac{\partial^{2} W}{\partial \bar{Z}^{2}}=\rho_{\mathrm{R}} \frac{\partial^{2} W}{\partial t^{2}}$

where $Z=x+i y$ and $\bar{Z}=x-i y$ are a complex variable and its complex conjugate, respectively.

To express the analytical solution of wave fields around the tunnel, the conforming mapping method of a complex function is employed as follows:

$Z=\Omega(\zeta)=c\left(\zeta+\sum_{m=0}^{N} c_{m} \zeta^{-m}\right), \zeta=\xi+i \eta=\rho e^{i \theta}$

where $c$ and $c_{\mathrm{m}}$ are coefficients of the complex function; $\xi$ and $\eta$ are the rectangular coordinate axes in the $\zeta$ plane; $\rho$ and $\theta$ denote the polar coordinates in the $\zeta$ plane; and $i^{2}=-1$. The mapping function transforms the boundaries $L_{1}$ and $L_{2}$ (Fig. 1a) in the $Z$ plane into two concentric circles $S_{1}$ and $S_{2}$ (Fig. 1b) with $R_{2}(<1)$ and unit radius $\left(R_{1}\right)$ in the $\zeta$ plane.

Submitting Eq. (3) into Eq. (2), the governing equation is written as follows: 


$$
\begin{aligned}
& \left(C_{55}-C_{44}+2 i C_{45}\right) \frac{1}{\Omega^{\prime}(\zeta)} \frac{\partial}{\partial \zeta}\left(\frac{1}{\Omega^{\prime}(\zeta)} \frac{\partial W}{\partial \zeta}\right)+2\left(C_{44}+C_{55}\right) \frac{1}{\Omega^{\prime}(\zeta)} \frac{\partial}{\partial \zeta}\left(\frac{1}{\left.\overline{\Omega^{\prime}(\bar{\zeta})} \frac{\partial W}{\partial \bar{\zeta}}\right) .}\right. \\
& +\left(C_{55}-C_{44}-2 i C_{45}\right) \frac{1}{\overline{\Omega^{\prime}(\bar{\zeta})}} \frac{\partial}{\partial \bar{\zeta}}\left(\frac{1}{\left.\overline{\Omega^{\prime}(\bar{\zeta})} \frac{\partial W}{\partial \bar{\zeta}}\right)=\rho_{\mathrm{R}} \frac{\partial^{2} W}{\partial t^{2}}}\right.
\end{aligned}
$$

Additional variables on the complex function in Eq. (4) are introduced as:

$$
\left.\begin{array}{l}
\chi=\frac{1}{2}[(1-i \gamma) Z+(1+i \gamma) \bar{Z}] \\
\bar{\chi}=\frac{1}{2}[(1-i \bar{\gamma}) Z+(1+i \bar{\gamma}) \bar{Z}]
\end{array}\right\}
$$

where $\gamma$ is a complex constant and is determined by $\gamma=-C_{45} / C_{44}+i \sqrt{C_{55} C_{44}-C_{45}^{2}} / C_{44}$.

Then, considering the steady solution, i.e. $W=w e^{-i \omega t}$ ( $\omega$ is circular frequency of the SH wave; $w$ is the anti-plane deformation ignoring the time variable $t$ ), and submitting Eq. (5) into Eq. (4), the governing equation in the $\zeta$ plane is rewritten as follows:

$$
\frac{\partial^{2} w}{\partial \chi \partial \bar{\chi}}=\left(\frac{i K_{\mathrm{I}}}{2}\right)^{2} w
$$

where $K_{\mathrm{I}}=\omega / C_{\mathrm{I}}, C_{\mathrm{I}}=\mu_{\mathrm{I}} / \rho_{\mathrm{R}}, \mu_{\mathrm{I}}=\left(C_{44} C_{55}-C_{45}^{2}\right) / C_{44}$. It is noted that the time-dependent term $e^{-i \omega t}$ of the incident wave and the following wave fields is omitted.

\section{Wave fields and stresses in the rock mass and tunnel lining}

\subsection{Wave fields and stresses in the anisotropic rock mass}

(1) Incident wave: The anti-plane SH wave with incident angle $\alpha$ is expanded as complex form in the $\zeta$ plane as follows (Liu, 1988):

$$
w^{(\mathrm{i} n)}=w_{0} \sum_{n=-\infty}^{\infty} i^{n} J_{n}\left(K_{a}|\Omega(\zeta)|\right)\left[\frac{\Omega(\zeta)}{|\Omega(\zeta)|}\right]^{n} e^{-i n \alpha}
$$

where $w_{0}$ denotes an amplitude of the incident $\mathrm{SH}$ wave; $K_{\mathrm{a}}$ is the wave number in the rock mass determined by $\omega=K_{\mathrm{a}} C_{\mathrm{a}}$ with $C_{a}=\left[\left(C_{55} \cos ^{2} \alpha+2 C_{45} \sin \alpha \cos \alpha+C_{44} \sin ^{2} \alpha\right) / \rho_{R}\right]^{\frac{1}{2}}$; and $J_{\mathrm{n}}(\cdot)$ is the $n$th Bessel function of the first kind.

The stresses resulting from the incident wave are expressed as Eqs. (8) and (9). 


$$
\begin{aligned}
& \tau_{r z, \mathrm{I}}^{(i n)}=\frac{C_{55} K_{\mathrm{a}} w_{0}}{4}\left\{\begin{array}{l}
{\left[\begin{array}{l}
(1+\beta) \sum_{n=-\infty}^{\infty} i^{n} J_{n-1}\left(K_{\mathrm{a}}|\Omega(\zeta)|\right)\left[\frac{\Omega(\zeta)}{|\Omega(\zeta)|}\right]^{n-1} e^{-i n \alpha}- \\
(1-\beta-2 i \kappa) \sum_{n=-\infty}^{\infty} i^{n} J_{n+1}\left(K_{\mathrm{a}}|\Omega(\zeta)|\left[\frac{\Omega(\zeta)}{|\Omega(\zeta)|}\right]^{n+1} e^{-i n \alpha}\right]
\end{array}\right] \begin{array}{l}
\frac{\zeta}{R} \frac{\Omega^{\prime}(\zeta)}{\Omega^{\prime}(\zeta) \mid}+ \\
(1-\beta+2 i \kappa) \sum_{n=-\infty}^{\infty} i^{n} J_{n-1}\left(K_{\mathrm{a}}|\Omega(\zeta)|\left[\frac{\Omega(\zeta)}{|\Omega(\zeta)|}\right]^{n-1} e^{-i n \alpha}-\right. \\
(1+\beta) \sum_{n=-\infty}^{\infty} i^{n} J_{n+1}\left(K_{\mathrm{a}}|\Omega(\zeta)|\right)\left[\frac{\Omega(\zeta)}{|\Omega(\zeta)|}\right]^{n+1} e^{-i n \alpha} \frac{\Omega^{\prime}(\bar{\zeta})}{\Omega^{\prime}(\zeta) \mid}
\end{array}}
\end{array}\right\}
\end{aligned}
$$

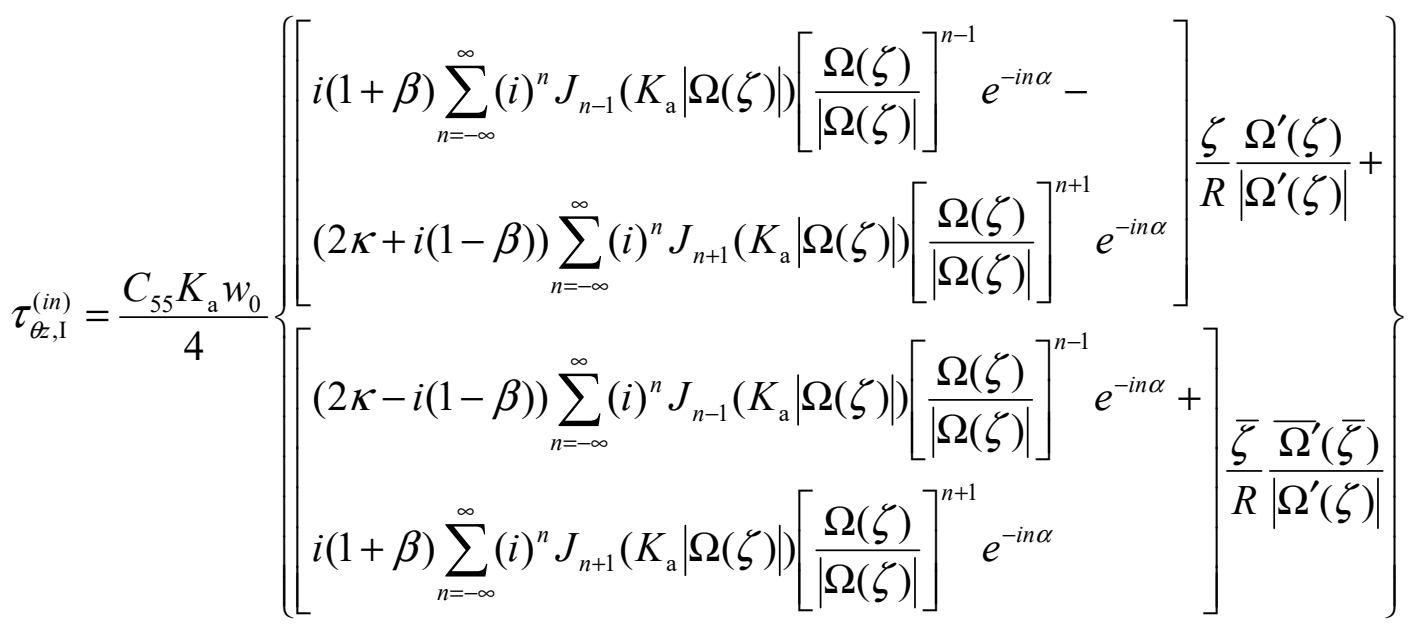

where $\beta=C_{44} / C_{55}$ and $\kappa=C_{45} / C_{55}$, representing the degree of rock anisotropy. It is assumed that $C_{44}$ is less than $C_{55}$, that is $\beta<1$. Then, with a higher $\kappa$ and smaller $\beta$, the medium has a higher degree of rock anisotropy. Since the elastic parameters $C_{55}, C_{44}, C_{45}$ satisfy the positive definite condition of the elastic matric, $\beta$ and $\kappa$ should meet condition of $\beta-\kappa^{2}>0$.

(2) Scattered wave: Following Eq. (6), the scattered wave around the tunnel lining is written as follows:

$$
w_{\mathrm{I}}^{(s c)}=\sum_{n=-\infty}^{\infty} A_{n} H_{n}^{(1)}\left(K_{\mathrm{I}}|\chi(\zeta)|\left[\frac{\chi(\zeta)}{|\chi(\zeta)|}\right]^{n}\right.
$$

where $A_{n}$ is an uncertain coefficient of the scattered wave around tunnel lining; $H_{\mathrm{n}}{ }^{(1)}(\cdot)$ is the Hankel function of the first kind and the $n$th order denoting the outgoing wave. The stresses resulting from the scattered wave are expressed as Eqs. (11) and (12). 


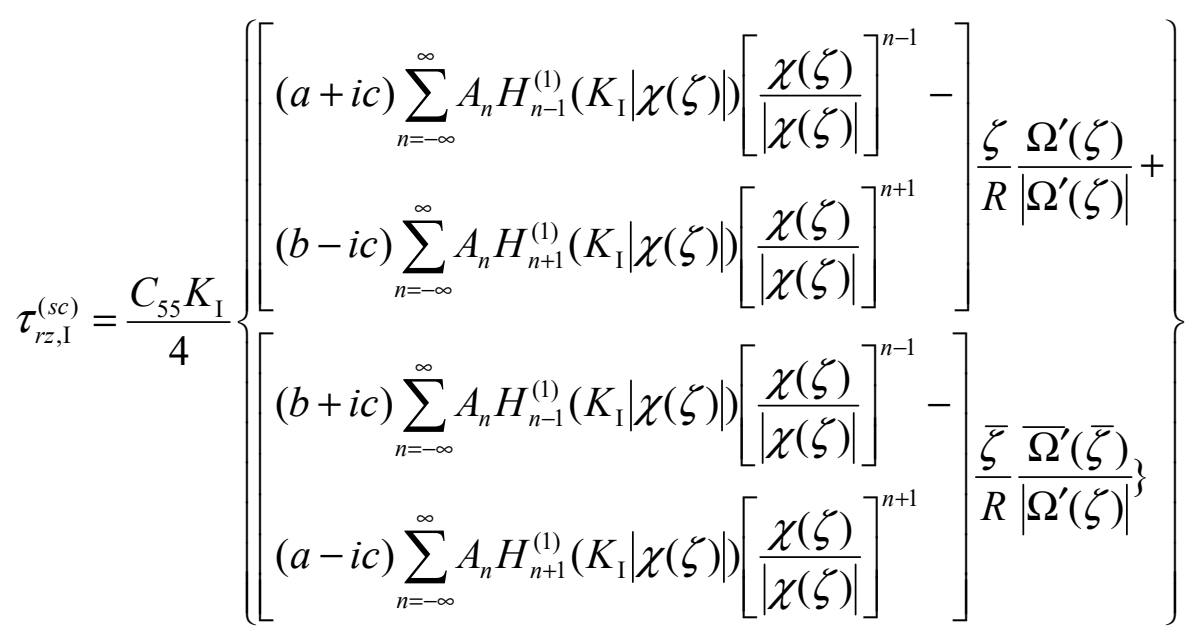

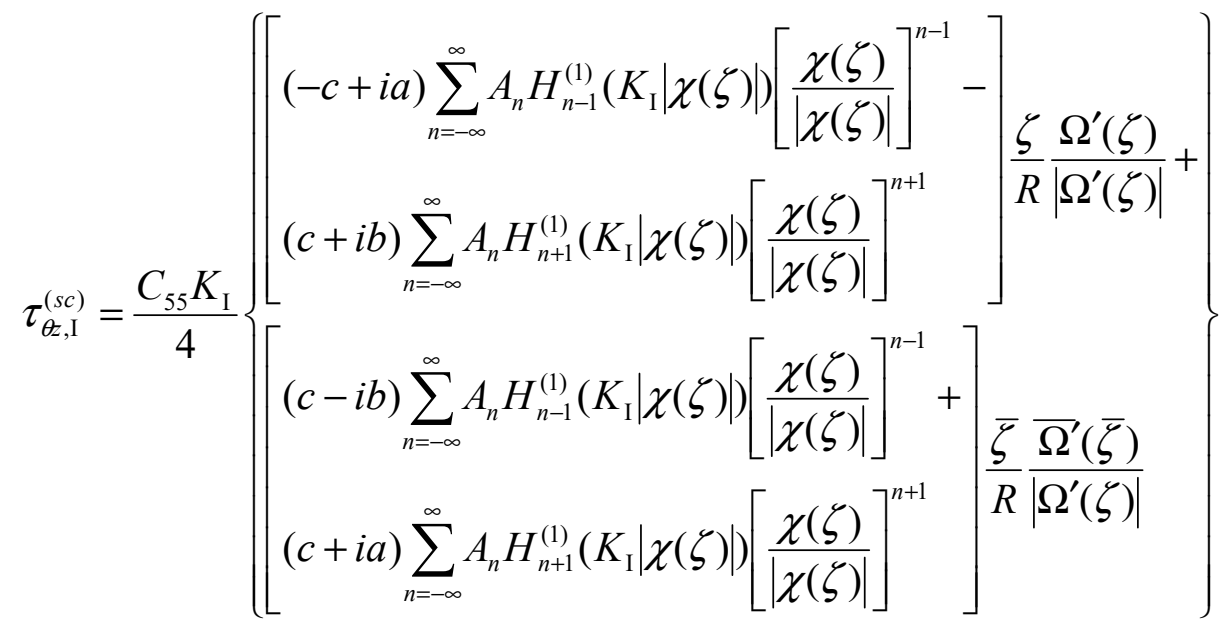

where $a=\sqrt{C_{55} C_{44}-C_{45}^{2}}\left(1+\sqrt{C_{55} C_{44}-C_{45}^{2}} / C_{44}\right), b=-\sqrt{C_{55} C_{44}-C_{45}^{2}}\left(1-\sqrt{C_{55} C_{44}-C_{45}^{2}} / C_{44}\right)$ and $c=C_{45} \sqrt{C_{55} C_{44}-C_{45}^{2}} / C_{44}$.

\subsection{Wave fields and stresses in the isotropic tunnel lining}

(1)Reflected wave: The reflected wave that propagates outwards from the inner boundary of the isotropic tunnel lining is expressed as follows:

$$
w_{\mathrm{II}}{ }^{(r f)}=\sum_{n=-\infty}^{\infty} B_{n} H_{n}^{(1)}\left(K_{\mathrm{II}}|\Omega(\zeta)|\left[\frac{\Omega(\zeta)}{|\Omega(\zeta)|}\right]^{n}\right.
$$

where $B_{n}$ is an uncertain coefficient of the reflected wave in the tunnel lining and $K_{\text {II }}$ is the wave number in the tunnel lining determined by $\omega=K_{\mathrm{II}} C_{L}$ with $C_{L}=\sqrt{u_{L} / \rho_{L}}$.

The stresses resulting from the reflected wave are expressed as Eqs. (14) and (15). 


$$
\begin{aligned}
& \tau_{r z, \mathrm{II}}^{(r f)}=\frac{\mu_{L} K_{\mathrm{II}}}{2}\left\{\begin{array}{l}
\sum_{n=-\infty}^{\infty} B_{n} H_{n-1}^{(1)}\left(K_{\mathrm{II}}|\Omega(\zeta)|\left[\frac{\Omega(\zeta)}{|\Omega(\zeta)|}\right]^{n-1} \frac{\zeta}{R} \frac{\Omega^{\prime}(\zeta)}{\Omega^{\prime}(\zeta) \mid}-\right. \\
\sum_{n=-\infty}^{\infty} B_{n} H_{n+1}^{(1)}\left(K_{\mathrm{II}}|\Omega(\zeta)|\right)\left[\frac{\Omega(\zeta)}{|\Omega(\zeta)|}\right]^{n+1} \frac{\bar{\zeta}}{\frac{\Omega^{\prime}(\zeta)}{R}} \frac{\Omega^{\prime}(\zeta) \mid}{}
\end{array}\right\} \\
& \tau_{\theta z, \mathrm{II}}^{(r f)}=\frac{i \mu_{L} K_{\mathrm{II}}}{2}\left\{\begin{array}{l}
\sum_{n=-\infty}^{\infty} B_{n} H_{n-1}^{(1)}\left(K_{\mathrm{II}}|\Omega(\zeta)|\right)\left[\frac{\Omega(\zeta)}{|\Omega(\zeta)|}\right]^{n-1} \frac{\zeta}{R} \frac{\Omega^{\prime}(\zeta)}{\left|\Omega^{\prime}(\zeta)\right|}+ \\
\sum_{n=-\infty}^{\infty} B_{n} H_{n+1}^{(1)}\left(K_{\mathrm{II}}|\Omega(\zeta)|\right)\left[\frac{\Omega(\zeta)}{|\Omega(\zeta)|}\right]^{n+1} \frac{\bar{\zeta}}{R} \frac{\overline{\Omega^{\prime}(\bar{\zeta})}}{\left|\Omega^{\prime}(\zeta)\right|}
\end{array}\right\}
\end{aligned}
$$

(2) Refracted wave: The refracted wave being confined into the tunnel lining is expressed as follows:

$$
w_{\mathrm{II}}{ }^{(r r)}=\sum_{n=-\infty}^{\infty} C_{n} H_{n}^{(2)}\left(K_{\mathrm{II}}|\Omega(\zeta)|\left[\frac{\Omega(\zeta)}{\mid \Omega(\zeta)}\right]^{n}\right.
$$

where $C_{\mathrm{n}}$ is an uncertain coefficient of the refracted wave in the tunnel lining; $H_{\mathrm{n}}{ }^{(2)}(\cdot)$ is the Hankel function of the second kind and the $n$th order denoting the ingoing wave.

The stresses resulting from the refracted wave are written as Eqs. (17) and (18):

$$
\begin{aligned}
& \tau_{r z, \mathrm{II}}^{(r r)}=\frac{\mu_{L} K_{\mathrm{II}}}{2}\left\{\begin{array}{l}
\sum_{n=-\infty}^{\infty} C_{n} H_{n-1}^{(2)}\left(K_{\mathrm{II}}|\Omega(\zeta)|\right)\left[\frac{\Omega(\zeta)}{\mid \Omega(\zeta)}\right]^{n-1} \frac{\zeta}{R} \frac{\Omega^{\prime}(\zeta)}{\left|\Omega^{\prime}(\zeta)\right|}- \\
\sum_{n=-\infty}^{\infty} C_{n} H_{n+1}^{(2)}\left(K_{\mathrm{II}}|\Omega(\zeta)|\left[\frac{\Omega(\zeta)}{|\Omega(\zeta)|}\right]^{n+1} \frac{\bar{\zeta}}{\frac{\Omega^{\prime}(\zeta)}{R}} \frac{\Omega^{\prime}(\zeta) \mid}{}\right.
\end{array}\right\} \\
& \tau_{\theta z, \mathrm{II}}^{(r r)}=\frac{i \mu_{L} K_{\mathrm{II}}}{2}\left\{\begin{array}{l}
\sum_{n=-\infty}^{\infty} C_{n} H_{n-1}^{(2)}\left(K_{\mathrm{II}}|\Omega(\zeta)|\right)\left[\frac{\Omega(\zeta)}{|\Omega(\zeta)|}\right]^{n-1} \frac{\zeta}{R} \frac{\Omega^{\prime}(\zeta)}{\left|\Omega^{\prime}(\zeta)\right|}+ \\
\sum_{n=-\infty}^{\infty} C_{n} H_{n+1}^{(2)}\left(K_{\mathrm{II}}|\Omega(\zeta)|\right)\left[\frac{\Omega(\zeta)}{|\Omega(\zeta)|}\right]^{n+1} \frac{\bar{\zeta}}{R} \frac{\overline{\Omega^{\prime}(\zeta)}}{\left|\Omega^{\prime}(\zeta)\right|}
\end{array}\right\}
\end{aligned}
$$

\section{Boundary condition}

In the anisotropic rock mass, the total wave fields are produced by the superposition of the incident wave and the scattered wave expressed as Eq. (19).

$$
w_{\mathrm{I}}^{(t)}=w^{(i n)}+w_{\mathrm{I}}^{(s c)}
$$

In the isotropic tunnel lining, the total wave fields are produced by the superposition of the 
refracted wave and reflected wave expressed as Eq. (20).

$$
w_{\mathrm{II}}^{(t)}=w_{\mathrm{II}}^{(r r)}+w_{\mathrm{II}}^{(r f)}
$$

An elastic model is introduced to analyze the imperfect interface effect. Its validity has been verified through theoretical (Yi et al., 2014; Fang et al., 2015, 2016a) and experimental results (Honarvar et al., 1998), as well as numerical simulation (Lombard and Piraux, 2006). With the elastic model, tractions at the outer boundary $\left(R=R_{1}, L_{1}\right.$ in Fig. 1a) of the tunnel lining are continuous, but displacements are discontinuous across the interface, described as follows:

$$
\left.\begin{array}{l}
\left.w_{\mathrm{I}}^{(t)}\right|_{R=R_{1}}-\left.w_{\mathrm{II}}^{(t)}\right|_{R=R_{1}}=\left.\frac{\tau_{r z, \mathrm{I}}^{(t)}}{k}\right|_{R=R_{1}} \\
\left.\tau_{r z, \mathrm{I}}^{(t)}\right|_{R=R_{1}}=\left.\tau_{r z, \mathrm{II}}^{(t)}\right|_{R=R_{1}}
\end{array}\right\}
$$

where $k$ is the stiffness of the imperfect interface, $\tau_{r z, \mathrm{I}}^{(t)}=\tau_{r z, \mathrm{I}}^{(i n)}+\tau_{r z, \mathrm{I}}^{(s c)}$ and $\tau_{r z, \mathrm{II}}^{(t)}=\tau_{r z, \mathrm{II}}^{(r r)}+\tau_{r z, \mathrm{II}}^{(r f)}$.

At the inner boundary $\left(R=R_{2}, L_{2}\right.$ in Fig. 1a) of the tunnel lining, tractions are free as expressed in Eq. (22).

$\left.\tau_{r z, \mathrm{II}}^{(t)}\right|_{R=R_{2}}=0$

where $\tau_{r z, \mathrm{II}}^{(t)}=\tau_{r z, \mathrm{II}}^{(r r)}+\tau_{r z, \mathrm{II}}^{(r f)}$.

Substituting Eqs. (19) and (20) into Eqs. (21) and (22), we obtain

$\sum_{m=1}^{3} \sum_{n=-\infty}^{\infty} K_{n}^{m j} X_{n}^{m}=Q^{j} \quad(j=1,2,3)$

where $X_{n}^{1}=\mathrm{A}_{n}, X_{n}^{2}=\mathrm{B}_{n}, X_{n}^{3}=\mathrm{C}_{n}, K_{n}^{m j}$ and $Q^{j}$ are presented in Appendix A.

Then multiplying both sides of Eq. (23) with the orthogonality of $e^{-i s \theta}(s=0, \pm 1, \pm 2, \pm 3 \ldots)$ and integrating over the interval $[-\pi, \pi]$, the unknown coefficients $A_{\mathrm{n}}, B_{\mathrm{n}}, C_{\mathrm{n}}$ can be determined straightforwardly by solving a set of infinite linear algebraic system with the expression as follows:

$\sum_{m=1}^{3} \sum_{n=-\infty}^{\infty} K_{n s}^{m j} X_{n}^{m}=Q_{s}^{j} \quad(j=1,2,3)$

where $K_{n s}^{m j}=\frac{1}{2 \pi} \int_{-\pi}^{\pi} K_{n}^{m j} e^{-i s \theta} d \theta, Q_{s}^{j}=\frac{1}{2 \pi} \int_{-\pi}^{\pi} Q^{j} e^{-i s \theta} d \theta$. In the following analysis, the truncation number is tested to be 5 for the low frequency, whereas 7 for the high frequency. 
Liu also pointed out that a smaller truncated number could be adopted when the wave frequency is smaller (Liu and Wang, 2012; Liu et al., 2013).

\section{Numerical example and discussion}

To analyze the effects of anisotropy and imperfect interface on the dynamic response around a deep underground tunnel subjected to anti-plane SH wave, the dimensionless dynamic stress concentration factor (DSCF) is introduced. According to the definition of DSCF by Pao and Mao (1973), DSCF of the circumferential stress around the tunnel is written as follows:

$D S C F=\tau_{\theta z, p}^{*}=\left|\frac{\tau_{\theta z, p}^{(\mathrm{t})}}{\tau_{0}}\right| \quad(p=\mathrm{I}$ for the rock mass and $p=\mathrm{II}$ for the tunnel lining $)$

where $\tau_{0}$ is maximum magnitude of the incident stress and defined as $\tau_{0}=C_{55} K_{a} w_{0}$; and $\tau_{\theta z, \mathrm{I}}^{(t)}=\tau_{\theta z, \mathrm{I}}^{(i n)}+\tau_{\theta z, \mathrm{I}}^{(s c)}, \tau_{\theta z, \mathrm{II}}^{(t)}=\tau_{\theta z, \mathrm{II}}^{(r r)}+\tau_{\theta z, \mathrm{II}}^{(r f)}$. The circumferential stress along the interface of the anisotropic rock mass and the tunnel lining could be found in Appendix B.

The detailed properties of the rock mass and tunnel lining, representatively encountered in the Tawarayama tunnel in Kumamoto Prefecture, Japan, are listed in Table 1. The Tawarayama tunnel runs through three different formations: the Quaternary Holocene, the Quaternary Pleistocene and the Tertiary Pliocene. Most of the tunnel is excavated in the Andesite lava with class $\mathrm{D}_{\text {I }}$ (Zhang et al., 2018). Here, the elastic constant $C_{55}$ is assumed to be equal to the elastic modulus of the Andesite lava (Shi et al., 1996). The following dimensionless variables and quantities are chosen for computation: the incident wave number is $K_{\mathrm{a}}{ }^{*}=K_{\mathrm{a}} R_{2}$, the interface stiffness parameter is $k^{*}=k R_{2} / C_{55}$. A lower dimensionless wave number $K_{\mathrm{a}}{ }^{*}=0.1$ and a higher dimensionless wave number $K_{\mathrm{a}}{ }^{*}=1.0$ are taken into consideration, which are the same as those in the work of Fang et al (2016b). Three sets of stiffness parameter are selected and they are $k^{*}=50.0,5.0$ and 0.5 , respectively. In addition, coefficients in the conformal mapping function (Eq. (3)) for the horseshoe-shaped tunnel lining are depicted in Table 2. Fig. 2 shows the mapping result and the actual dimension of the horseshoe cross-section in Tawarayama tunnel. The inner radius of the crown is $4.8 \mathrm{~m}$, and the thickness of the tunnel lining is $0.50 \mathrm{~m}$. 
Table 1 Input data for the material properties of the model

\begin{tabular}{ccc|cccc}
\hline Properties of rock mass & & \multicolumn{4}{|c}{ Properties of tunnel lining } \\
\hline$E_{R}(\mathrm{Gpa})$ & $v$ & $\rho_{R}\left(\mathrm{~kg} / \mathrm{m}^{3}\right)$ & $E_{L}(\mathrm{Gpa})$ & $v$ & $\rho_{L}\left(\mathrm{~kg} / \mathrm{m}^{3}\right)$ & $t(\mathrm{~m})$ \\
\hline 13.08 & 0.3 & 2200 & 22.00 & 0.2 & 2450 & 0.50 \\
\hline
\end{tabular}

Note: $E_{\mathrm{R}}$ denotes elastic modulus of the rock mass.

$E_{\mathrm{L}}$ denotes elastic modulus of the tunnel lining.

$t$ denotes thickness of the tunnel lining.

Table 2 Coefficients in conformal mapping function

\begin{tabular}{ccccccccc}
\hline$R_{1}(\mathrm{~m})$ & $R_{2}(\mathrm{~m})$ & $\bar{\rho}$ & $c_{1}$ & $c_{2}$ & $c_{3}$ & $c_{4}$ & $c_{5}$ & $c_{6}$ \\
\hline 5.3 & 4.8 & 0.93 & 0.00240 & $-0.04721 i$ & -0.04766 & $0.02038 i$ & -0.00333 & $0.00977 i$ \\
\hline
\end{tabular}

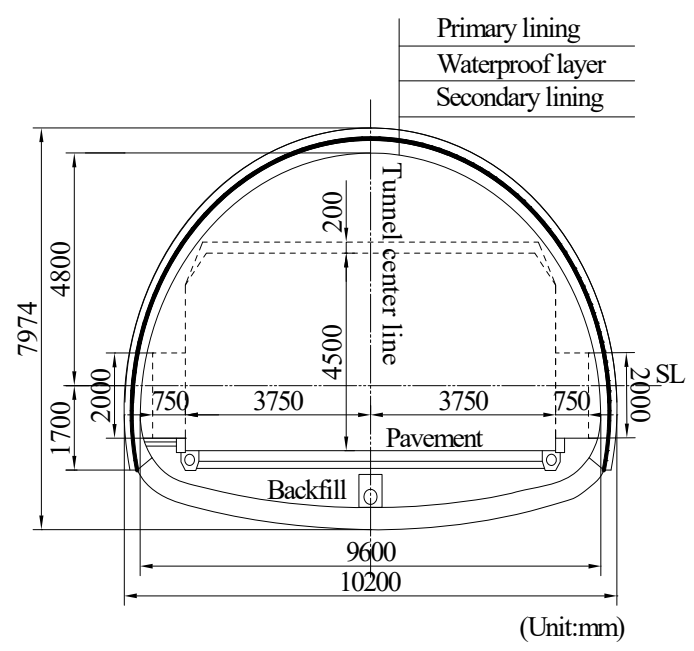

Figure 2 Mapping cross-section (red dotted line) for the horseshoe cross-section (black line) in Tawarayama tunnel.

To validate this dynamic model, comparisons with the existing approaches are presented in Fig. 3 In this figure, rock mass is isotropic, the interface is perfect, and the tunnel lining is circular. By defining $\beta=1.0, \kappa=0.0$, rock mass is reduced to be isotropic. After trial-and-error, the dimensionless stiffness $k^{*}=10^{4}$ is sufficient to guarantee the interface perfect approximately with the present parameters. The reduced model is consistent with that by Fang (2016a). Since distributions of the dynamic stresses around the circular tunnel lining are perfectly symmetrical about the incident direction, the maximum dynamic stresses $D S C F_{\text {max }}$ upper and $D S C F_{\text {max_lower }}$ near both ends of the vertical line of the incident direction are taken into consideration in the following analysis, as illustrated in Fig. 4. Two types of anisotropy degree, i.e. lower degree of anisotropy (LDA) with $\beta=0.8, \kappa=0.2$ and higher degree of anisotropy (HDA) with $\beta=0.5, \kappa=0.5$, are employed. 

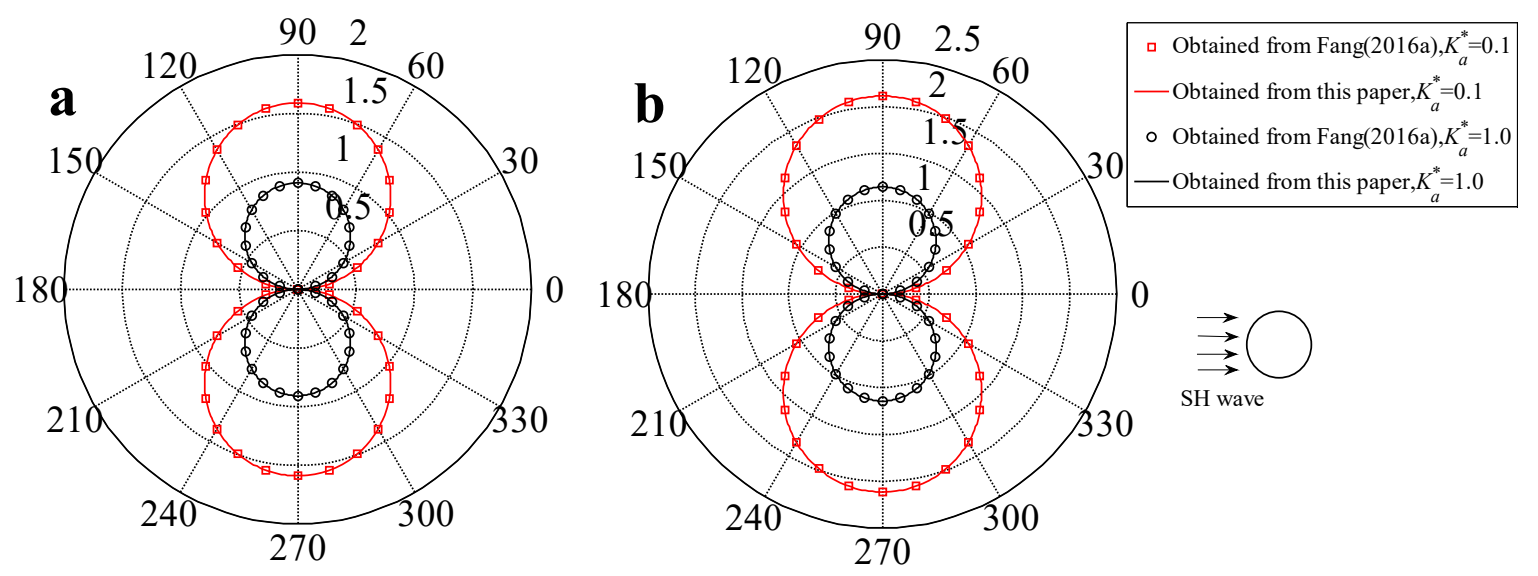

Figure 3 Comparisons of the present solutions with those by Fang (2016a) for circular tunnel under horizontal SH-wave in the isotropic rock mass. (a) DSCF of the inner side of the tunnel lining; (b) DSCF of the surrounding rock mass at the interface.
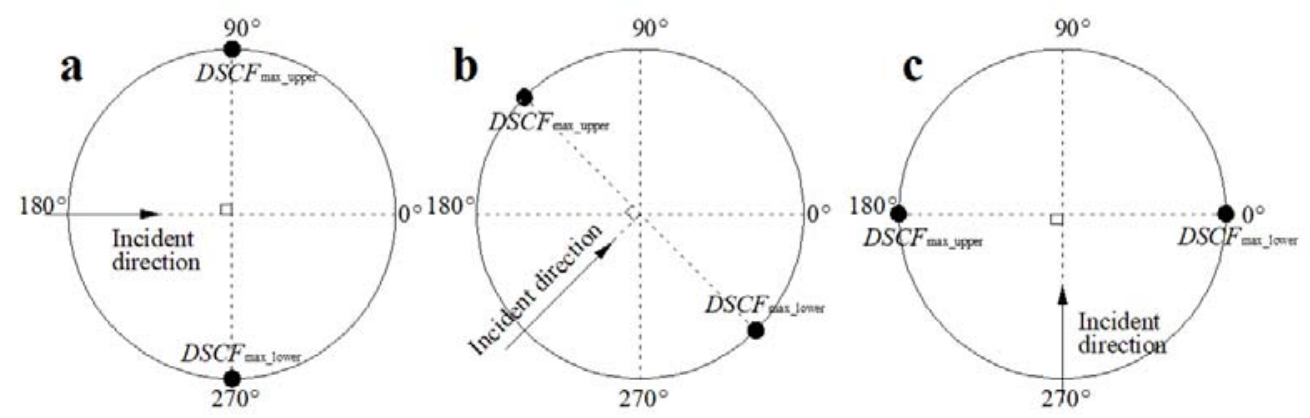

Figure 4 Schematic of the monitoring area of the tunnel lining subjected to horizontal incident wave (a), inclined incident wave (b) and vertical incident wave (c).

Figs. 5 7 present effect of the anisotropy parameters on the circumferential stresses in the lining and medium with different incident wave. When rock mass is isotropic $(\beta=1.0, \kappa=$ 0.0), distributions of the DSCF are bilaterally symmetric (the black line in Fig. 5). Similar with the circular tunnel (Fig. 3), the maximum dynamic stresses of the tunnel lining occur at the position $\theta=90^{\circ}\left(D S C F_{\text {max_upper }}\right)$ and $\theta=270^{\circ}\left(D S C F_{\text {max_lower }}\right)$. When rock mass becomes anisotropic $(\beta \neq 1.0, \kappa \neq 0.0)$, distributions of the DSCF become asymmetric (the blue and red lines in Fig. 5). In the LDA rock mass, the maximum dynamic stresses of the tunnel lining occur at the position $\theta=94^{\circ}\left(D S C F_{\text {max_upper }}\right)$ and $\theta=278^{\circ}\left(D S C F_{\text {max_lower }}\right)$. In the HDA rock mass, the maximum dynamic stresses of the tunnel lining occur at the position $\theta=98^{\circ}$ $\left(D S C F_{\text {max } \_ \text {upper }}\right)$ and $\theta=286^{\circ}\left(D S C F_{\text {max_lower }}\right)$. For the cases of inclined and vertical incident SH wave (Figs. 6 and 7), distributions of the DSCF in the anisotropic rock mass also become 
asymmetric by comparison with those in the isotropic rock mass. Corresponding maximum dynamic stress occurrence positions are listed in Table 3, where it is observed that the maximum dynamic stress positions of both the anisotropic cases differ from those of the isotropic one, and the dynamic stress redistribution is related to the degree of rock anisotropy, especially in the lining above the spring line $\left(\theta\right.$ from $0^{\circ}$ to $\left.180^{\circ}\right)$. The conclusion can be drawn that rock anisotropy exerts an important influence on the dynamic stress distribution around the tunnel lining. Moreover, the case with HDA $(\beta=0.5, \kappa=0.5)$ exhibits much larger value due to the larger difference of rock characteristics in different directions compared with the LDA case $(\beta=0.5, \kappa=0.2)$.
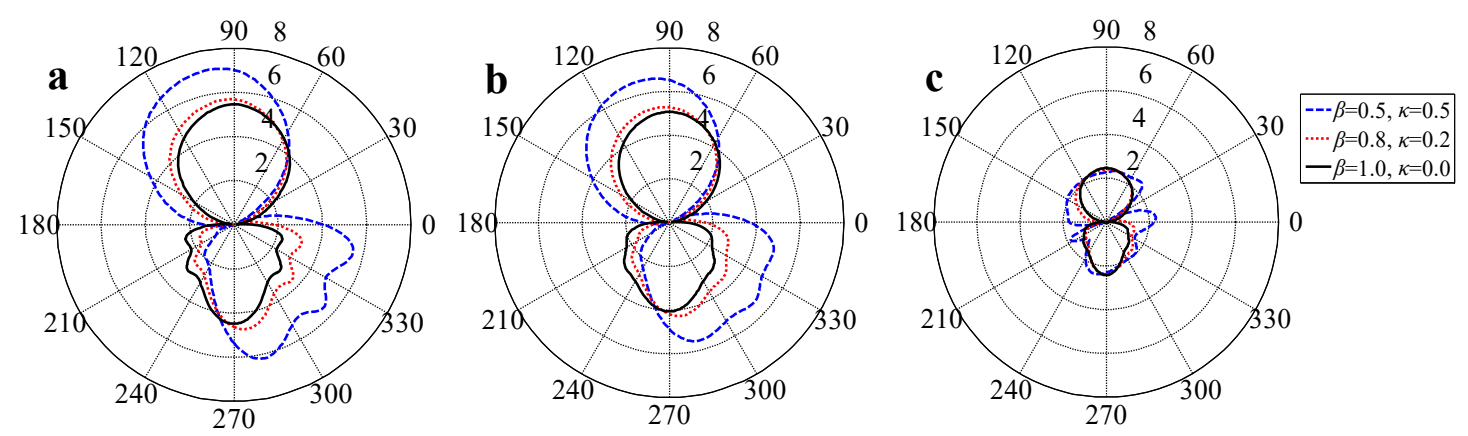

Figure 5 Effect of anisotropy on the DSCF of the tunnel lining and rock mass $\left(\alpha=0^{\circ}, K_{a}{ }^{*}=0.1\right)$. (a) DSCF at the inner side of the tunnel lining; (b) DSCF at the outer side of the tunnel lining; (c) DSCF of the

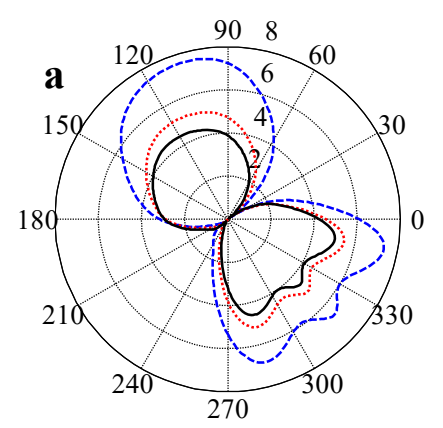
surrounding rock mass at the interface.
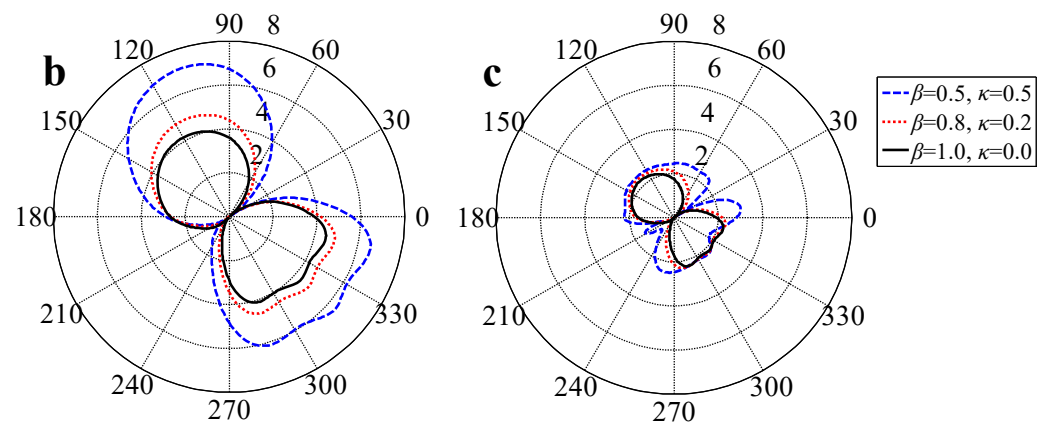

Figure 6 Effect of anisotropy on the DSCF of the tunnel lining and rock mass $\left(\alpha=45^{\circ}, K_{a}{ }^{*}=0.1\right)$. (a) DSCF at the inner side of the tunnel lining; (b) DSCF at the outer side of the tunnel lining; (c) DSCF of the surrounding rock mass at the interface. 

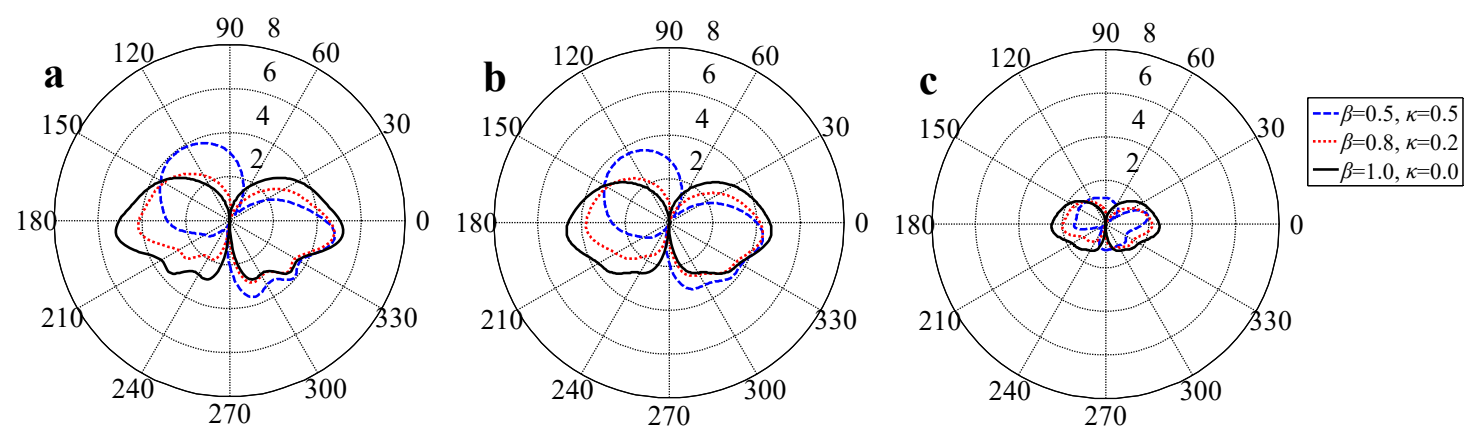

Figure 7 Effect of anisotropy on the DSCF of the tunnel lining and rock mass $\left(\alpha=90^{\circ}, K_{a}{ }^{*}=0.1\right)$. (a)

DSCF at the inner side of the tunnel lining; (b) DSCF at the outer side of the tunnel lining; (c) DSCF of the surrounding rock mass at the interface.

Table 3 also lists the maximum of the DSCF near the monitoring area (Fig. 4) in the anisotropic and isotropic rock mass. When the wave is excited vertically, maximums of the DSCF around the tunnel lining in the anisotropic rock mass are smaller than those in the isotropic one. While, when the tunnel is subjected to horizontal or inclined seismic wave, maximums of the DSCF around the tunnel lining are larger than those in the isotropic one. An explanation is that rock anisotropy contributes to different propagation characteristics in each direction of the rock mass. Furthermore, for the cases of horizontal and inclined wave, a much larger maximum can be obtained by choosing a much higher degree of anisotropy.

Table 3 Maximum of DSCF in the anisotropic and isotropic rock mass

\begin{tabular}{|c|c|c|c|c|c|c|c|}
\hline $\begin{array}{l}\text { Incident } \\
\text { angle }\end{array}$ & $\begin{array}{c}\text { Rock } \\
\text { anisotropy }\end{array}$ & $\begin{array}{c}D S C F_{\text {max }} \text { upper } \\
\left(L_{1} \text { side }\right)\end{array}$ & $\begin{array}{c}D S C F_{\text {max }} \text { lower } \\
\left(L_{1} \text { side }\right)\end{array}$ & $\begin{array}{c}D S C F_{\text {max }} \text { upper } \\
\left(L_{2} \text { side }\right)\end{array}$ & $\begin{array}{c}D S C F_{\text {max lower }} \\
\left(L_{2} \text { side }\right)\end{array}$ & $\theta_{\max \_ \text {upper }} /^{\circ}$ & $\theta_{\max \_l o w e r} 1^{\circ}$ \\
\hline \multirow{3}{*}{$\alpha=0^{\circ}$} & $\beta=0.5, \kappa=0.5$ & 6.63 & 5.57 & 7.12 & 6.20 & 98 & 286 \\
\hline & $\beta=0.8, \kappa=0.2$ & 5.29 & 4.30 & 5.70 & 4.76 & 94 & 278 \\
\hline & $\beta=1.0, \kappa=0.0$ & 5.07 & 4.06 & 5.46 & 4.48 & 90 & 270 \\
\hline \multirow{3}{*}{$\alpha=45^{\circ}$} & $\beta=0.5, \kappa=0.5$ & 7.10 & 6.40 & 7.57 & 6.64 & 107 & 320 \\
\hline & $\beta=0.8, \kappa=0.2$ & 4.86 & 4.82 & 5.17 & 5.00 & 116 & 319 \\
\hline & $\beta=1.0, \kappa=0.0$ & 4.17 & 4.36 & 4.44 & 4.53 & 123 & 320 \\
\hline \multirow{3}{*}{$\alpha=90^{\circ}$} & $\beta=0.5, \kappa=0.5$ & 3.68 & 4.35 & 3.93 & 4.86 & 129 & 352 \\
\hline & $\beta=0.8, \kappa=0.2$ & 3.80 & 4.32 & 4.18 & 4.81 & 183 & 354 \\
\hline & $\beta=1.0, \kappa=0.0$ & 4.70 & 4.70 & 5.21 & 5.21 & 187 & 355 \\
\hline
\end{tabular}

Figs. 8 and 9 illustrate effect of imperfect interface on dynamic response of horseshoe-shaped tunnel under vertical incident wave with a lower frequency $\left(K_{\mathrm{a}}{ }^{*}=0.1\right)$. In a similar fashion to the circular tunnel, the maximum dynamic stresses around the tunnel occur at the position near the top of the vault and the bottom of the invert. The interface effect at these positions is the greatest and increasing interface stiffness contributes to the dynamic stress decreasing. 
Near the positions $\theta=0^{\circ}$ and $\theta=180^{\circ}$, the interface effect is not obvious on the dynamic stress. By comparing Figs. 8 and 9, it can be observed that a much higher degree of rock anisotropy brings an increase of the interface effect, especially at the invert.
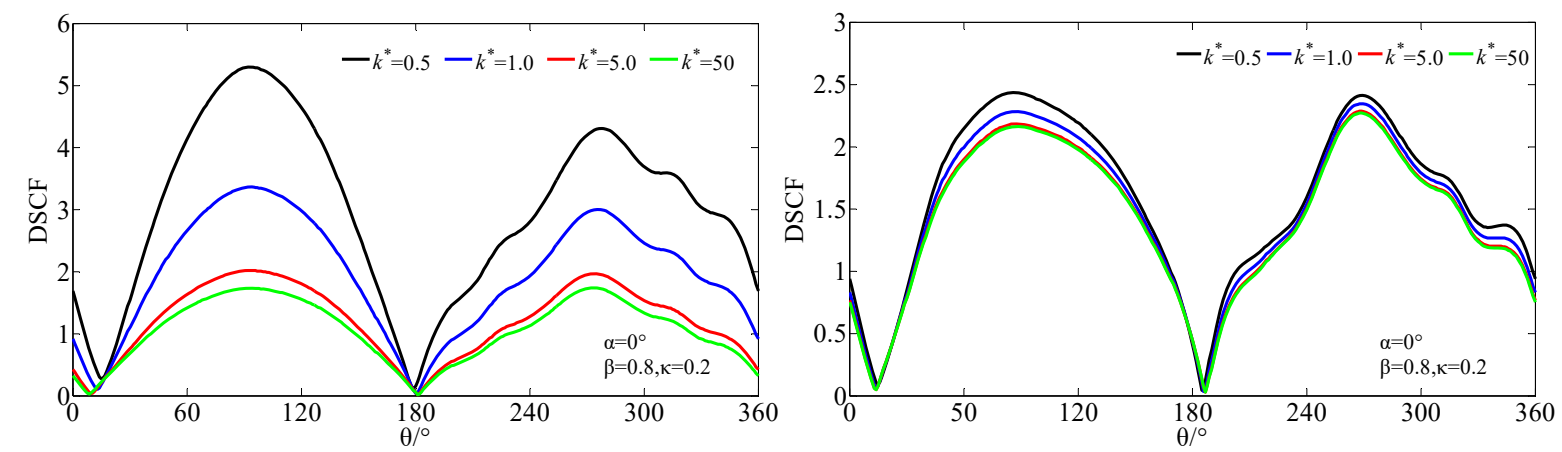

Figure 8 Effect of interface stiffness on DSCF of the outer side of tunnel lining (a) and rock mass at the interface (b). $\left(K_{a}{ }^{*}=0.1, \beta=0.8, \kappa=0.2\right)$.
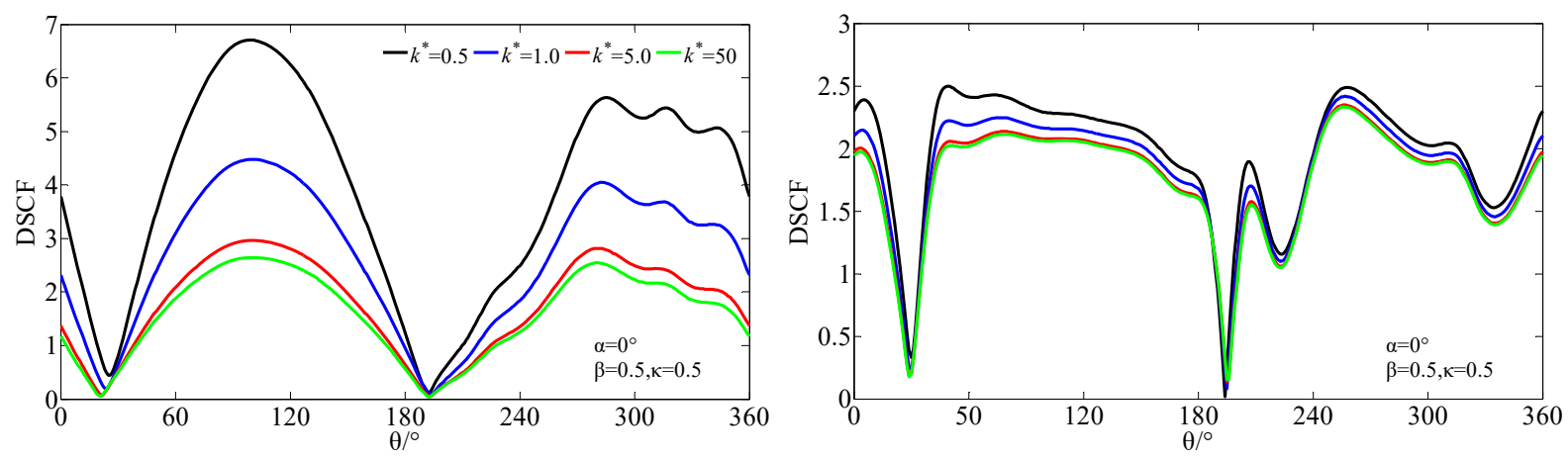

Figure 9 Effect of interface stiffness on DSCF of the outer side of tunnel lining (a) and rock mass at the

$$
\text { interface (b). }\left(K_{a}^{*}=0.1, \beta=0.5, \kappa=0.5\right)
$$

Figs. 10 and 11 illustrate effect of imperfect interface on dynamic response of horseshoe-shaped tunnel under vertical incident wave with a higher frequency $\left(K_{\mathrm{a}}{ }^{*}=1.0\right)$. Dynamic stresses become a bit more complicated with several peaks due to the high frequency loading and the rock anisotropy. In comparison with the results in the region of low frequency, the interface effect on the dynamic stress increases significantly. By comparing Figs. $8 \sim 11$, the interface effect is significant if the $k^{*}$ is less than 1.0, as observed by Fang et al. (2016a). Furthermore, the interface effect increases with anisotropic characteristic of rock mass. 

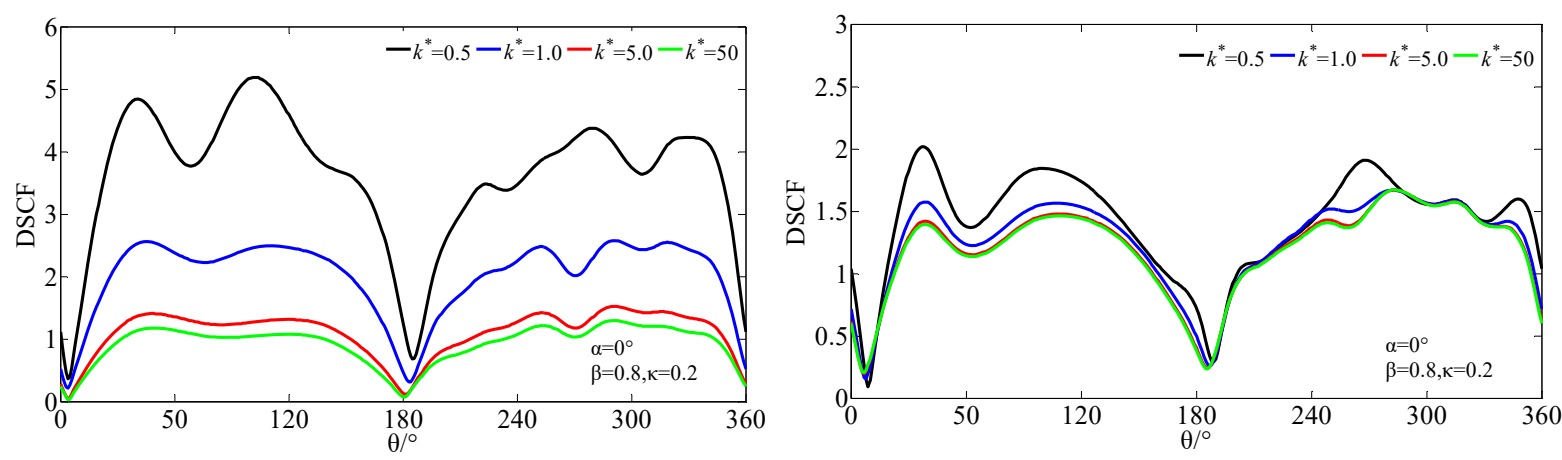

Figure 10 Effect of interface stiffness on DSCF of the outer side of tunnel lining (a) and rock mass at the interface (b). $\left(K_{a}{ }^{*}=1.0, \beta=0.8, \kappa=0.2\right)$.
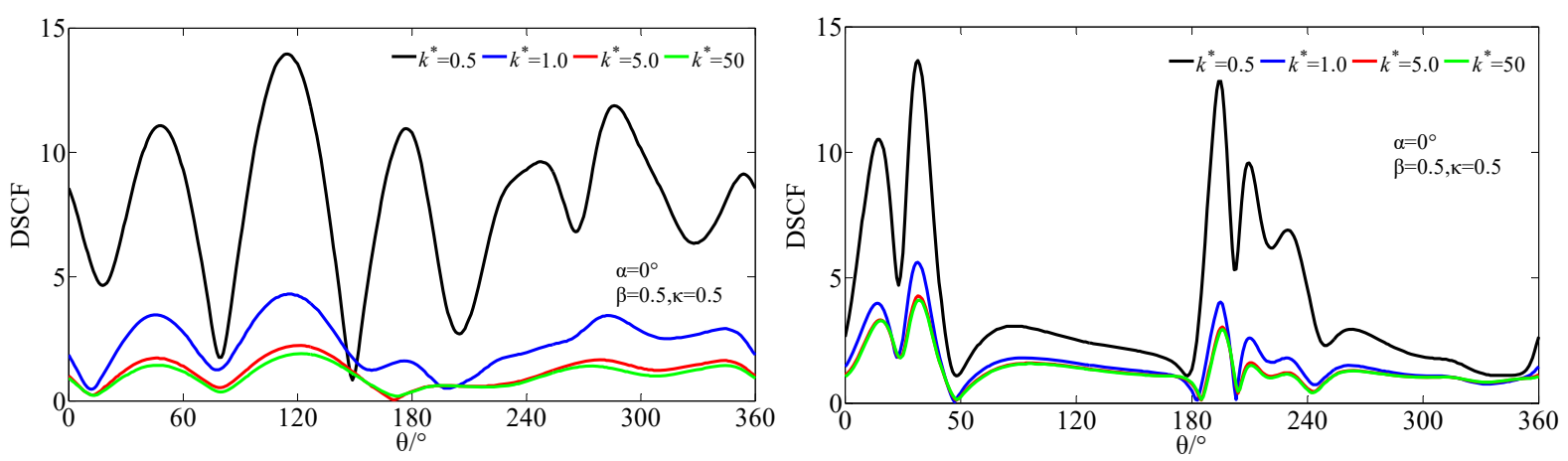

Figure 11 Effect of interface stiffness on DSCF of the outer side of tunnel lining (a) and rock mass at the interface (b). $\left(K_{a}{ }^{*}=1.0, \beta=0.5, \kappa=0.5\right)$.

The DSCF variations of tunnel lining at $\theta=45^{\circ}$ (spandrel), $\theta=90^{\circ}$ (vault) and $\theta=270^{\circ}$ (invert) with the interface stiffness $k^{*}$ are presented in Fig. 12. The DSCFs on the vault, spandrel and invert are high when the stiffness is low. Increasing interface stiffness contributes to decreasing DSCF. As the stiffness increases, the dynamic stress concentration decreases greatly to a steady value. Taking Fig. 12a as an illustration, the decreasing percentages at the vault and invert of the lining inner side are $65.1 \%$ and $56.6 \%$ with the interface stiffness increasing from 0.5 to 10 , while the decreasing percentages are $6.8 \%$ and $5.4 \%$ with interface stiffness increasing from 10 to 50 . It can be concluded that the effect of interface stiffness decreases gradually along with the interface stiffness increasing. Thus, with a much larger interface stiffness, the interface effect can be neglected to make the interface perfect. This observation also corroborates the selection of $k^{*}$ in the section of validation of the present analytical method. 


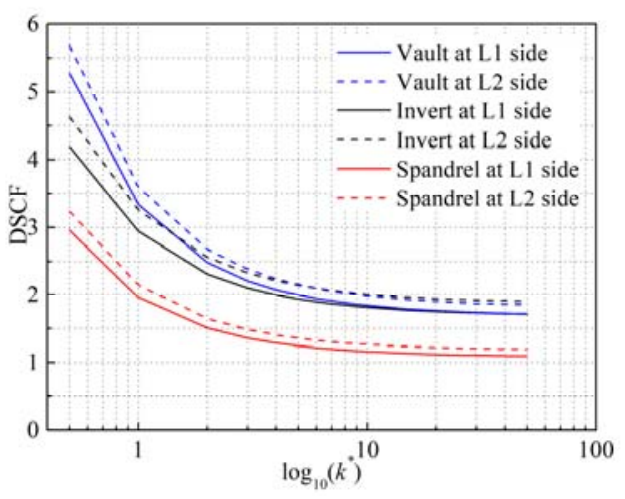

(a)

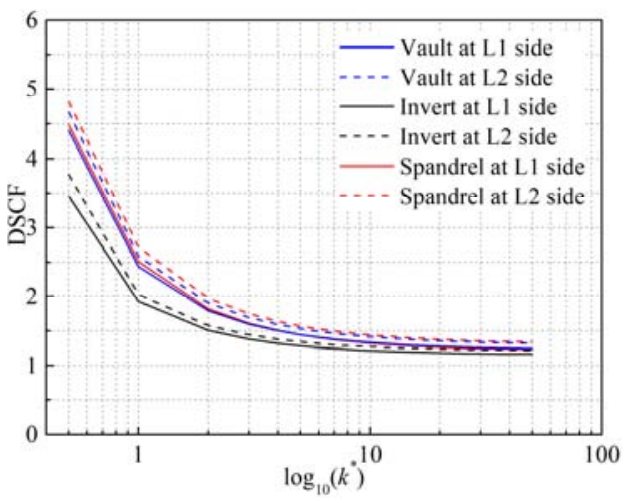

(b)

Figure 12 Variation of DSFC along with the interface stiffness constants $k^{*}(\beta=0.8, \kappa=0.2)$. (a) $K_{a}{ }^{*}=0.1$;

(b) $K_{a}^{*}=1.0$.

\section{Conclusions}

Combining with the wave function expansion method, the complex function and mapping function, the dynamic response of a horseshoe-shaped tunnel with imperfect interface around the tunnel lining in an anisotropic rock mass is theoretically presented under an incidence of an anti-plane SH wave. The elastic spring interface model is used to present the imperfect interface. A series of analytical results are illustrated to study the effect of rock anisotropy and imperfect interface on the dynamic stress concentration of the tunnel lining and its surrounding rock mass. Some important conclusions are as follows.

The rock anisotropy is of significant importance on the scattering of a seismic wave. The rock anisotropy changes the shape of DSCF distribution, unlike the symmetric distribution in the isotropic rock mass. And a case with HDA exhibits much more serious asymmetry than that with LDA. Besides, different propagation characteristics in each direction due to the rock anisotropy contributes to a different DSCF maximum variation with the rock anisotropy.

Increasing interface stiffness contributes to a decreasing DSCF. When high frequency wave is excited, the shapes of DSCF become a bit more complicated with several peaks, compared with the smooth shape under a low frequency incident wave. And the effect of the interface between the tunnel lining and its surrounding rock mass weakens gradually along with the interface stiffness increasing. While, a much higher degree of rock anisotropy brings an increase of the interface effect, especially at the invert. 


\section{Acknowledgement}

This work was funded by National Natural Science Foundation of China (No. 51379117), Provincial Natural Science Foundation of Shandong Province, China (No. ZR2017PEE018), China Scholarship Council (CSC No. 201508370077), JSPS Grant-in-Aid for Scientific Research (Number 17H03506), and JSPS-NSFC Bilateral Joint Research Project, Japan. The authors appreciate the contribution of Prof. Raymond L. Sterling from Louisiana Technology University in the writing of this paper.

\section{References}

Allred, B., Daniels, J.J., Ehsani, M.R. 2008. Handbook of agricultural geophysics. CRC Press.

Bewick R.P., Kaiser P.K., 2009, Influence of rock mass anisotropy on tunnel stability. ROCKENG09, Proceedings of the 3rd CANUS Rock Mechanics Symposium, Toronto, Ont. Edited by Diederichs and Grasselli. Paper (No. 3995).

Chen Z.G., 2012, Dynamic stress concentration around shallow cylindrical cavity by SH wave in anisotropically elastic half-space. Rock Soil Mech. 33(3), 264-270. (in Chinese)

Chen Z.G., 2015, Effect of shallow buried cavity on anti-plane motion of ground surface in anisotropic half-space. Acta Seismologica Sinica. 37(4), 617-628. (in Chinese)

Davis C.A., Lee V.W., Bardet J.P., 2001, Transverse response of underground cavities and pipes to incident SV waves. Earthq. Eng. Struct. D. 30(3), 383-410.

Dravinski M., Yu C.W., 2011, Peak surface motion due to scattering of plane harmonic P, SV, or Rayleigh waves by a rough cavity embedded in an elastic half-space. J. Seismol. 15, $131-45$.

Duan, K., Wu, W., Kwok, C.Y., 2018. Discrete element modelling of stress-induced instability of directional drilling boreholes in anisotropic rock. Tunn. Undergr. Sp. Tech. 81, 55-67.

Fang X.Q., Jin H.X., Liu J.X., Huang M.J., 2016a, Imperfect bonding effect on dynamic response of a non-circular lined tunnel subjected to shear waves. Tunn. Undergr. Sp. Tech. $56,226-231$.

Fang X.Q., Jin H.X., Wang B.L., 2015, Dynamic interaction of two circular lined tunnels with imperfect interfaces under cylindrical P-waves. Int. J. Rock Mech. Min. Sci. 79, 172-182. 
Fang X.Q., Jin H.X., 2016b, Visco-elastic imperfect bonding effect on dynamic response of a non-circular lined tunnel subjected to P and SV waves. Soil Dyn. Earthq. Eng. 88, 1-7.

Fang X.Q., Jin H.X., 2017, Dynamic response of a non-circular lined tunnel with visco-elastic imperfect interface in the saturated poroelastic medium. Comput. Geotec. 83, 98-105.

Gatmiri B., Eslami H., 2007, Scattering of harmonic waves by a circular cavity in a porous medium: complex functions theory approach. Int. J. Geomech. 7(5), 371-381.

Ren G., Smith J.V., Tang J.W., Xie Y.M., 2005, Underground excavation shape optimization using an evolutionary procedure. Compu. Geotech. 32(2), 122-132.

Han F., Liu D.K., 1997, Scattering of plane SH-wave on semi-canyon topography of arbitrary shape with lining in anisotropic media. Appl. Math. Mech. 18(8), 753-761. (In Chinese)

Honarvar F., Sinclair A.N., 1998, Nondestructive evaluation of cylindrical components by resonance acoustic spectroscopy. Ultrasonics. 36(8), 845-854.

Lin C.H., Lee V.W., Todorovska M.I., Trifunac M.D., 2010, Zero-stress, cylindrical wave functions around a circular underground tunnel in a flat, elastic half-space: Incident P-waves. Soil Dyn. Earthq. Eng. 30(10), 879-894.

Liu D.K., 1988, Dynamic stress concentration around a circular hole due to SH-wave in anisotropic media. Acta Mech. Sinica. 4(2),146-155.

Liu Q., Wang R., 2012, Dynamic response of twin closely-spaced circular tunnels to harmonic plane waves in a full space. Tunn. Undergr. Sp. Tech. 32, 212-220.

Liu Q., Zhang C., Todorovska M.I., 2016, Scattering of SH waves by a shallow rectangular cavity in an elastic half space. Soil Dyn. Earthq. Eng. 90, 147-157.

Liu Q., Zhao M., Wang L., 2013, Scattering of plane P, SV or Rayleigh waves by a shallow lined tunnel in an elastic half space. Soil Dyn. Earthq. Eng. 49, 52-63.

Lombard B., Piraux J., 2006, Numerical modeling of elastic waves across imperfect contacts. SIAM J. Sci. Comput. 28(1), 172-205.

Pao Y.H., Mow C.C., Diffraction of elastic waves and dynamic stress concentrations. New York: Crane, Russak \& Co. Inc.. 1973.

Shen Y., Gao B., Yang X., Tao S., 2014, Seismic damage mechanism and dynamic deformation characteristic analysis of mountain tunnel after Wenchuan earthquake. Eng. Geol. 180, 85-98. 
Shi S.X., Han F., Wang Z.Q., Liu D.K., 1996, The interaction of Plane SH-wave and non-circular cavity surfaced with lining in anisotropic media. Appl. Math. Mech. 17(9), 809-820. (in Chinese)

Smerzini C., Aviles J., Paolucci R., Sánchez-Sesma F.J., 2009, Effect of underground cavities on surface earthquake ground motion under SH wave propagation. Earthq. Eng. Struct. Dyn. 38(12), 1441-1460.

Son M., Cording E.J., 2007, Ground-lining interaction in rock tunneling. Tunn. undergr. Sp. Tech. 22(1), 1-9.

Wang J.H., Zhou X.L., Lu J.F., 2005, Dynamic stress concentration around elliptic cavities in saturated poroelastic soil under harmonic plane waves. Int. J. solids Struct. 42(14), 4295-4310.

Wang T.T., Hsu J.T., Chen C.H., Huang T.H., 2014, Response of a tunnel in double-layer rocks subjected to harmonic P-and S-waves. Int. J. Rock Mech. Min. Sci. 70, 435-443.

Wu D., Gao B., Shen Y., Zhou J., Chen G., 2015, Damage evolution of tunnel portal during the longitudinal propagation of Rayleigh waves. Nat. Hazards. 75(3), 2519-2543.

Yi C., Zhang P., Johansson D., Nyberg U., 2014, Dynamic response of a circular lined tunnel with an imperfect interface subjected to cylindrical P-waves. Comput. Geotech. 55, $165-171$.

Yu C.W., Dravinski M., 2010, Scattering of plane harmonic P, SV and Rayleigh waves by a completely embedded corrugated elastic inclusion. Wave Motion. 47,156-67.

Yu H., Chen J., Bobet A., Yuan Y., 2016, Damage observation and assessment of the Longxi tunnel during the Wenchuan earthquake. Tunn. Undergr. Sp. Tech. 54,102-116.

Zhang X.P., Jiang Y.J., Sugimoto S., 2018, Seismic damage assessment of mountain tunnel: a case study on the Tawarayama Tunnel due to the 2016 Kumamoto Earthquake. Tunn. Undergr. Sp. Tech. 71, 138-148.

Zimmerman C., Stern M., 1993, Boundary element solution of 3-D wave scatter problems in a poroelastic medium. Eng. Anal. Bound. Elem.12, 223-40. 


\section{Appendix A}

Coefficients $K_{n}^{i j}$ and $R^{j}$ in Eq. (23).

$$
\begin{aligned}
& K_{n}^{11}=H_{n}^{(1)}\left(K_{\mathrm{I}}\left|\chi\left(\zeta_{1}\right)\right|\left[\frac{\chi\left(\zeta_{1}\right)}{\left|\chi\left(\zeta_{1}\right)\right|}\right]^{n}\right.
\end{aligned}
$$

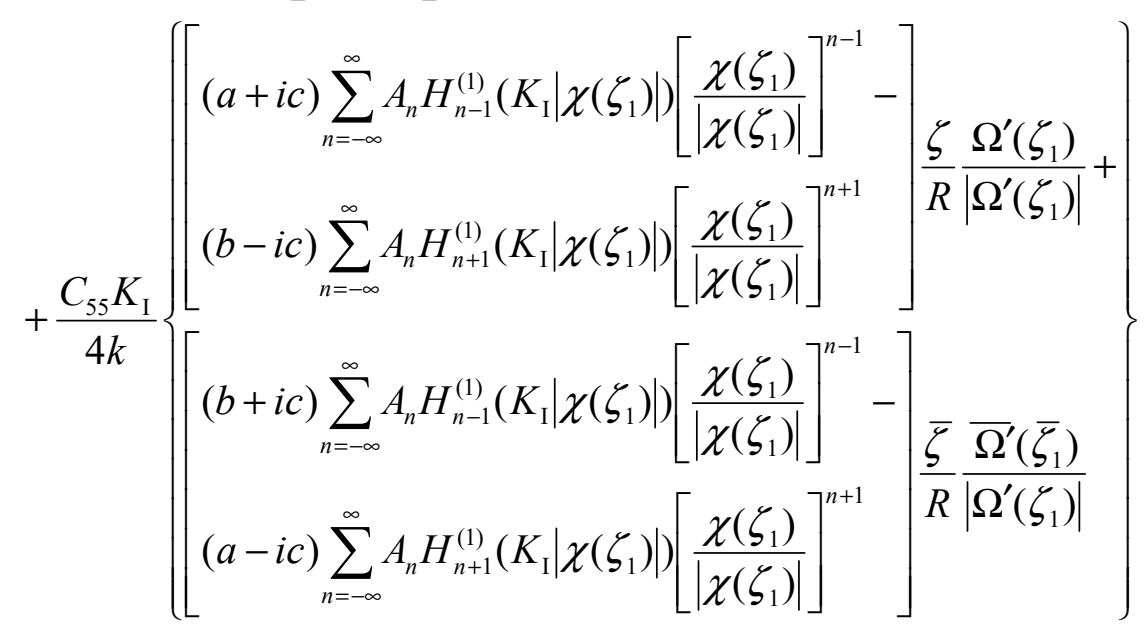

$$
\begin{aligned}
& K_{n}^{12}=-H_{n}^{(1)}\left(K_{\mathrm{II}}\left|\Omega\left(\zeta_{1}\right)\right|\right)\left[\frac{\Omega\left(\zeta_{1}\right)}{\Omega\left(\zeta_{1}\right) \mid}\right]^{n} \\
& K_{n}^{13}=-H_{n}^{(2)}\left(K_{\mathrm{II}}\left|\Omega\left(\zeta_{1}\right)\right|\right)\left[\frac{\Omega\left(\zeta_{1}\right)}{\left|\Omega\left(\zeta_{1}\right)\right|}\right]^{n}
\end{aligned}
$$

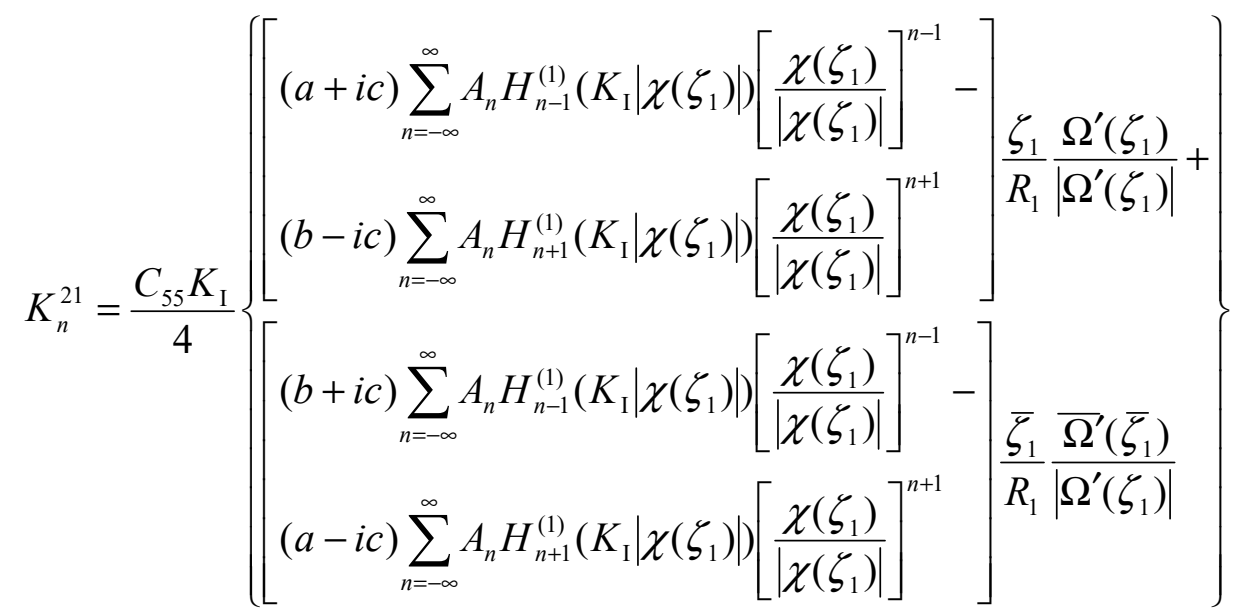

$K_{n}^{22}=\frac{\mu_{L} K_{\mathrm{II}}}{2}\left\{H_{n-1}^{(1)}\left(K_{\mathrm{II}}\left|\Omega\left(\zeta_{1}\right)\right|\left[\frac{\Omega\left(\zeta_{1}\right)}{\Omega\left(\zeta_{1}\right) \mid}\right]^{n-1} \frac{\zeta_{1}}{R_{1}} \frac{\Omega^{\prime}\left(\zeta_{1}\right)}{\Omega^{\prime}\left(\zeta_{1}\right)}-H_{n+1}^{(1)}\left(K_{\mathrm{II}}\left|\Omega\left(\zeta_{1}\right)\right|\left[\frac{\Omega\left(\zeta_{1}\right)}{\left|\Omega\left(\zeta_{1}\right)\right|}\right]^{n+1} \frac{\zeta_{1}}{R_{1}} \frac{\overline{\Omega^{\prime}}\left(\bar{\zeta}_{1}\right)}{\Omega^{\prime}\left(\zeta_{1}\right) \mid}\right\}\right.\right.$ 


$$
K_{n}^{23}=\frac{\mu_{L} K_{\mathrm{II}}}{2}\left\{H _ { n - 1 } ^ { ( 2 ) } \left(K_{\mathrm{II}}\left|\Omega\left(\zeta_{1}\right)\right|\left[\frac{\Omega\left(\zeta_{1}\right)}{\left|\Omega\left(\zeta_{1}\right)\right|}\right]^{n-1} \frac{\zeta_{1}}{R_{1}} \frac{\Omega^{\prime}\left(\zeta_{1}\right)}{\Omega^{\prime}\left(\zeta_{1}\right)}-H_{n+1}^{(2)}\left(K_{\mathrm{II}}\left|\Omega\left(\zeta_{1}\right)\right|\left[\left[\frac{\Omega\left(\zeta_{1}\right)}{\Omega\left(\zeta_{1}\right) \mid}\right]^{n+1} \frac{\overline{\zeta_{1}}}{R_{1}} \frac{\overline{\Omega^{\prime}}\left(\bar{\zeta}_{1}\right)}{\Omega^{\prime}\left(\zeta_{1}\right) \mid}\right\}\right.\right.\right.
$$

$$
K_{n}^{31}=0
$$

$$
K_{n}^{32}=\frac{\mu_{L} K_{\mathrm{II}}}{2}\left\{H_{n-1}^{(1)}\left(K_{\mathrm{II}}\left|\Omega\left(\zeta_{2}\right)\right|\left[\frac{\Omega\left(\zeta_{2}\right)}{\left|\Omega\left(\zeta_{2}\right)\right|}\right]^{n-1} \frac{\zeta_{2}}{R_{2}} \frac{\Omega^{\prime}\left(\zeta_{2}\right)}{\left|\Omega^{\prime}\left(\zeta_{2}\right)\right|}-H_{n+1}^{(1)}\left(K_{\mathrm{II}}\left|\Omega\left(\zeta_{2}\right)\right|\right)\left[\frac{\Omega\left(\zeta_{2}\right)}{\left|\Omega\left(\zeta_{2}\right)\right|}\right]^{n+1} \frac{\bar{\zeta}}{R_{2}} \frac{\overline{\Omega^{\prime}}\left(\bar{\zeta}_{2}\right)}{\Omega^{\prime}\left(\zeta_{2}\right) \mid}\right\}\right.
$$

$$
K_{n}^{33}=\frac{\mu_{L} K_{\mathrm{II}}}{2}\left\{H _ { n - 1 } ^ { ( 2 ) } \left(K_{\mathrm{II}}\left|\Omega\left(\zeta_{2}\right)\right|\left[\frac{\Omega\left(\zeta_{2}\right)}{\left|\Omega\left(\zeta_{2}\right)\right|}\right]^{n-1} \frac{\zeta_{2}}{R_{2}} \frac{\Omega^{\prime}\left(\zeta_{2}\right)}{\left|\Omega^{\prime}\left(\zeta_{2}\right)\right|}-H_{n+1}^{(2)}\left(K_{\mathrm{II}}\left|\Omega\left(\zeta_{2}\right)\right|\left[\frac{\Omega\left(\zeta_{2}\right)}{\left|\Omega\left(\zeta_{2}\right)\right|}\right]^{n+1} \frac{\overline{\zeta_{2}}}{R_{2}} \frac{\bar{\Omega}\left(\bar{\zeta}_{2}\right)}{\left|\Omega^{\prime}\left(\zeta_{2}\right)\right|}\right\}\right.\right.
$$

$$
\begin{aligned}
& Q^{1}=-w_{0} \sum_{n=-\infty}^{\infty} i^{n} J_{n}\left(K_{a}\left|\Omega\left(\zeta_{1}\right)\right|\left[\frac{\Omega\left(\zeta_{1}\right)}{\left|\Omega\left(\zeta_{1}\right)\right|}\right]^{n} e^{-i n \alpha}\right. \\
& \left.+\frac{C_{55} K_{a} w_{0}}{4 k}\left\{\begin{array}{l}
{\left[\begin{array}{l}
(1+\beta) \sum_{n=-\infty}^{\infty} i^{n} J_{n-1}\left(K_{a}\left|\Omega\left(\zeta_{1}\right)\right|\right. \\
(1-\beta-2 i \kappa) \sum_{n=-\infty}^{\infty} i^{n} J_{n+1}\left(K_{a}\left|\Omega\left(\zeta_{1}\right)\right|\right. \\
\left|\Omega\left(\zeta_{1}\right)\right|
\end{array}\right]^{n-1} e^{-i n \alpha}-} \\
{\left[\frac{\Omega\left(\zeta_{1}\right)}{\Omega\left(\zeta_{1}\right) \mid}\right]^{n+1} e^{-i n \alpha}}
\end{array}\right] \begin{array}{l}
\frac{\zeta_{1}}{R_{1}} \frac{\Omega^{\prime}\left(\zeta_{1}\right)}{\Omega^{\prime}\left(\zeta_{1}\right) \mid}+ \\
(1-\beta+2 i \kappa) \sum_{n=-\infty}^{\infty} i^{n} J_{n-1}\left(K_{a}\left|\Omega\left(\zeta_{1}\right)\right|\right)\left[\frac{\Omega\left(\zeta_{1}\right)}{\left|\Omega\left(\zeta_{1}\right)\right|}\right]^{n-1} e^{-i n \alpha}- \\
(1+\beta) \sum_{n=-\infty}^{\infty} i^{n} J_{n+1}\left(K_{a}\left|\Omega\left(\zeta_{1}\right)\right|\left[\frac{\Omega\left(\zeta_{1}\right)}{\left|\Omega\left(\zeta_{1}\right)\right|}\right]^{n+1} e^{-i n \alpha}\right.
\end{array}\right]
\end{aligned}
$$

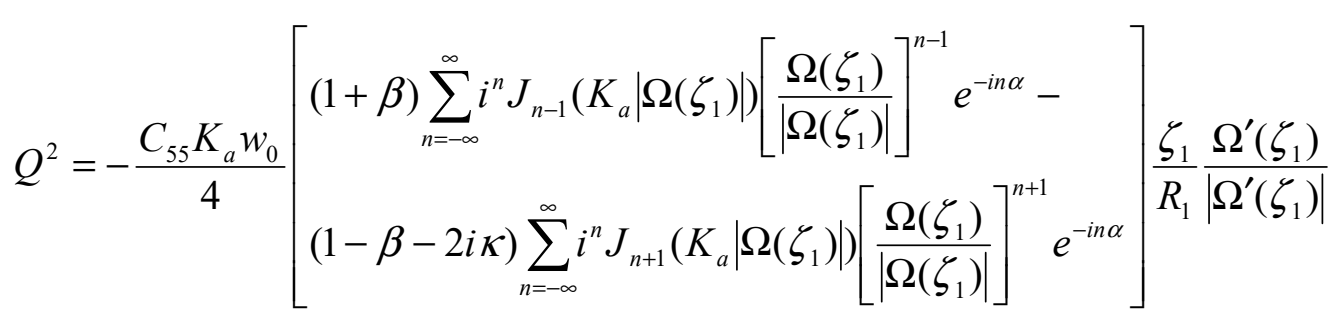

$$
\begin{aligned}
& +\frac{C_{55} K_{a} w_{0}}{4}\left[\begin{array}{l}
(1-\beta+2 i \kappa) \sum_{n=-\infty}^{\infty} i^{n} J_{n-1}\left(K_{a}\left|\Omega\left(\zeta_{1}\right)\right|\right)\left[\frac{\Omega\left(\zeta_{1}\right)}{\Omega\left(\zeta_{1}\right) \mid}\right]^{n-1} e^{-i n \alpha}- \\
(1+\beta) \sum_{n=-\infty}^{\infty} i^{n} J_{n+1}\left(K_{a}\left|\Omega\left(\zeta_{1}\right)\right|\left[\frac{\Omega\left(\zeta_{1}\right)}{\left|\Omega\left(\zeta_{1}\right)\right|}\right]^{n+1} e^{-i n \alpha}\right.
\end{array}\right] \frac{\overline{\zeta_{1}}}{R_{1} \frac{\overline{\Omega^{\prime}}\left(\bar{\zeta}_{1}\right)}{\Omega^{\prime}\left(\zeta_{1}\right) \mid}}
\end{aligned}
$$


$Q^{3}=0$

where, $\zeta_{1}=R_{1} e^{i \theta}, \zeta_{2}=R_{2} e^{i \theta}$.

\section{Appendix B}

The circumferential stress along the interface of the anisotropic rock mass is as follows:

$$
\begin{aligned}
& \tau_{\theta z, \mathrm{I}}^{(t)}=\frac{C_{55} K_{a} w_{0}}{4}\left\{\begin{array}{l}
{\left[\begin{array}{l}
i(1+\beta) \sum_{n=-\infty}^{\infty} i^{n} J_{n-1}\left(K_{a}\left|\Omega\left(\zeta_{1}\right)\right|\right)\left[\frac{\Omega\left(\zeta_{1}\right)}{\left|\Omega\left(\zeta_{1}\right)\right|}\right]^{n-1} \exp (-i n \alpha) \\
-i(1-\beta-2 i \kappa) \sum_{n=-\infty}^{\infty} i^{n} J_{n+1}\left(K_{a}\left|\Omega\left(\zeta_{1}\right)\right|\right)\left[\frac{\Omega\left(\zeta_{1}\right)}{\left|\Omega\left(\zeta_{1}\right)\right|}\right]^{n+1} \exp (-i n \alpha)
\end{array}\right]} \\
\frac{\zeta_{1}}{R_{1}} \frac{\Omega^{\prime}\left(\zeta_{1}\right)}{\Omega^{\prime}\left(\zeta_{1}\right) \mid}+ \\
\left.-i(1-\beta+2 i \kappa) \sum_{n=-\infty}^{\infty} i^{n} J_{n-1}\left(K_{a}\left|\Omega\left(\zeta_{1}\right)\right|\right)\left[\frac{\Omega\left(\zeta_{1}\right)}{\left|\Omega\left(\zeta_{1}\right)\right|}\right]^{n-1} \exp (-i n \alpha)\right] \\
+i(1+\beta) \sum_{n=-\infty}^{\infty} i^{n} J_{n+1}\left(K_{a}\left|\Omega\left(\zeta_{1}\right)\right|\right)\left[\frac{\Omega\left(\zeta_{1}\right)}{\left|\Omega\left(\zeta_{1}\right)\right|}\right]^{n+1} \exp (-i n \alpha)
\end{array}\right]
\end{aligned}
$$

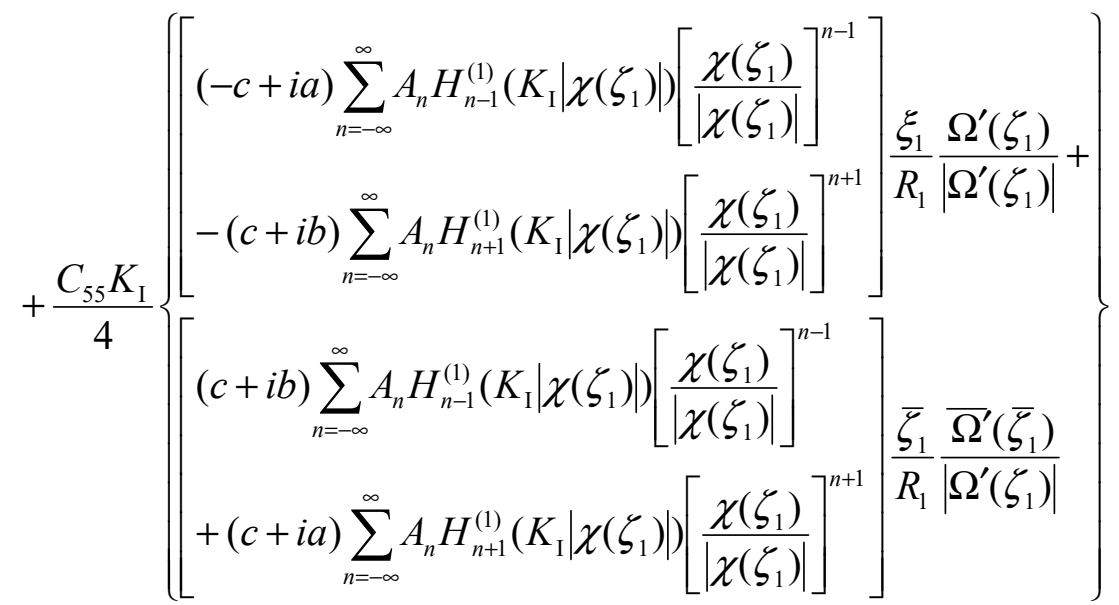

The circumferential stresses of outer $(g=1)$ and inner $(g=2)$ sides of the tunnel lining are written as follows:

$$
\begin{aligned}
\tau_{\theta z, \mathrm{II}}^{(t), g} & =\frac{i \mu_{L} K_{\mathrm{II}}}{2}\left\{\sum_{n=-\infty}^{\infty} B_{n} H_{n-1}^{(1)}\left(K_{\mathrm{II}}\left|\Omega\left(\zeta_{g}\right)\right|\right)\left[\frac{\Omega\left(\zeta_{g}\right)}{\mid \Omega\left(\zeta_{g}\right)}\right]^{n-1}+\sum_{n=-\infty}^{\infty} C_{n} H_{n-1}^{(2)}\left(K_{\mathrm{II}}\left|\Omega\left(\zeta_{g}\right)\right|\left[\frac{\Omega\left(\zeta_{g}\right)}{\left|\Omega\left(\zeta_{g}\right)\right|}\right]^{n-1}\right\} \frac{\zeta_{g}}{R_{g}} \frac{\Omega^{\prime}\left(\zeta_{g}\right)}{\left|\Omega^{\prime}\left(\xi_{g}\right)\right|}\right. \\
& +\frac{i \mu_{L} K_{\mathrm{II}}}{2}\left\{\sum_{n=-\infty}^{\infty} B_{n} H_{n+1}^{(1)}\left(K_{\mathrm{II}}\left|\Omega\left(\zeta_{g}\right)\right|\right)\left[\frac{\Omega\left(\zeta_{g}\right)}{\left|\Omega\left(\zeta_{g}\right)\right|}\right]^{n+1}+\sum_{n=-\infty}^{\infty} C_{n} H_{n+1}^{(2)}\left(K_{\mathrm{II}}\left|\Omega\left(\zeta_{g}\right)\right|\left[\frac{\Omega\left(\zeta_{g}\right)}{\left|\Omega\left(\zeta_{g}\right)\right|}\right]^{n+1}\right\} \frac{\zeta_{g}}{R_{g}} \frac{\overline{\Omega^{\prime}}\left(\bar{\zeta}_{g}\right)}{\left|\Omega^{\prime}\left(\zeta_{g}\right)\right|}\right.
\end{aligned}
$$

